\title{
Encadenamientos regionales en Colombia 2004-2012
}

\author{
Recibido: 21 de enero de 2016 - Aceptado: 21 de julio de 2016
}

Doi: dx.doi.org/10.12804/revistas.urosario.edu.co/economia/a.5240

\section{Lucas Wilfried Hahn-de-Castro*}

\section{Resumen}

Este trabajo analiza las relaciones económicas que existen entre los departamentos colombianos bajo un enfoque regional y sectorial. Se realiza para los años 2004 y 2012, períodos para los cuales existe una base de datos en formato insumo producto configurada durante el proceso de creación y posterior actualización del modelo multirregional CEER. Se utilizan dos metodologías: los indicadores obtenidos de la matriz inversa de Leontief y los indicadores de encadenamiento puro propuestos por Sonis et al. (1995). Los principales hallazgos del trabajo son 1) las regiones desarrolladas de Colombia muestran altos encadenamientos intrarregionales e interregionales hacia adelante, derivados de la matriz inversa de Leontief; 2) los sectores de industria y servicios, a pesar de haber perdido importancia, se mantienen como los de mayores encadenamientos productivos; 3) construcción y administración pública fueron los sectores de mayores incrementos; y 4) en el agregado, los encadenamientos departamentales no sufrieron cambios significativos.

Clasificación JEL: R12, R15.

Palabras clave: encadenamientos regionales, encadenamiento puro, matriz inversa de Leontief, modelo CEER.

* Economista del Centro de Estudios Económicos Regionales (CEER), Banco de la República, sucursal Cartagena. Correo electrónico: lhahndca@banrep.gov.co

Para citar este artículo: Hahn-de-Castro, L. W. (enero-junio, 2016). Encadenamientos regionales en Colombia 2004-2012. Revista de Economía del Rosario, 19(1), 29-56.

Doi:dx.doi.org/10.12804/revistas.urosario.edu.co/economia/a.5240 


\title{
Regional input-output linkages in Colombia 2004-2012
}

\begin{abstract}
This paper analyzes the economic linkages between the Colombian departments for the years 2004 and 2012 under a regional and sectorial approach, using the CEER Model data. Two methodologies are used: linkages derived from the Leontief inverse and the pure linkages approach proposed by Sonis et al. (1995). The main findings are 1) the more developed regions in Colombia have higher intraregional and interregional forward linkages; 2) the industry and service sectors remain the ones with the highest productive linkages, even after a period of declining relevance; 3 ) construction and public administration sectors had the largest increases; and 4) the regional linkages showed no significant changes in the national aggregate.
\end{abstract}

JEL classification: R12, R15.

Keywords: regional linkages, pure linkage approach, Leontief inverse matrix, CEER model.

\section{Encadeamentos regionais na Colômbia 2004-2012}

\section{Resumo}

Este trabalho analisa as relações económicas que existem entre os departamentos colombianos sob um enfoque regional e setorial. Realiza-se para os anos 2004 e 2012. Períodos para os quais existe uma base de dados em formato insumo produto configurada durante o processo de criação e posterior atualização do modelo multirregional CEER. Usam-se duas metodologias: os indicadores obtidos da matriz inversa de Leontief e os indicadores de encadeamento puro propostos por Sonis et al. (1995). Os principais resultados do trabalho são 1) as regiões desenvolvidas da Colômbia mostram altos encadeamentos intra-regionais e inter-regionais para adiante, derivados da matriz inversa de Leontief; 2) os setores de indústria e serviços, a pesar de ter perdido importância, se mantém como os de maiores encadeamentos produtivos; 3 ) construção e administração pública foram os setores de maiores incrementos; e 4) no agregado, os encadeamentos departamentais não sofreram mudanças significativas.

Classificação JEL: R12, R15.

Palavras-chave: encadeamentos regionais, encadeamento puro, matriz inversa de Leontief, modelo CEER. 


\section{Introducción}

El encadenamiento productivo, también llamado eslabonamiento, es una medida que permite determinar el impacto de un sector económico a otro. Se pueden encontrar dos tipos de encadenamiento: hacia adelante y hacia atrás. Para mostrar la diferencia entre ambos, se asume que la producción de un sector particular sube en una unidad. Con tal de producir esa unidad extra el sector demandará más insumos, por lo que la cantidad de bienes y servicios que la economía tiene que producir se incrementa. Este efecto se conoce como encadenamiento hacia atrás y se genera porque los sectores demandan insumos para producir sus propios bienes y servicios. Pero, a su vez, al aumentar su producción, ese sector está incrementando la cantidad de insumos que la economía tiene a su disposición para producir más bienes y servicios. Es decir, los sectores que puedan utilizar esa unidad extra en su proceso productivo tendrán una mayor disposición de recursos para poder desarrollar su actividad económica. Este es el encadenamiento hacia adelante y se da porque los sectores ofertan bienes y servicios que sirven como insumos para el resto de la economía.

Los encadenamientos suelen ser calculados para el agregado nacional, lo que permite identificar actividades económicas clave para el país. Sin embargo, también pueden ser calculados para distintas regiones de manera simultánea; es decir, los encadenamientos regionales. Utilizar un enfoque regional permite identificar dos tipos de efectos. El primero es el encadenamiento local o intrarregional del sector, que es el impacto que genera este sobre la economía de su propia región. El segundo es el encadenamiento interregional, que es la dependencia económica entre los sectores de distintas regiones.

El estudio de los encadenamientos productivos requiere de la construcción de matrices insumo producto. En este trabajo se utilizan los datos estimados durante la elaboración de dos modelos multirregionales, calibrados para los años 2004 y 2012 en conjunto por el Centro de Estudios Económicos Regionales (CEER) y la Fundación Instituto de Investigación Económica (FIPE, por su sigla en portugués) de la Universidad de Sao Paulo en Brasil. Las matrices regionales son un insumo para la estimación y actualización del modelo CEER, un modelo de equilibrio general computable que permite simular distintos escenarios de choque dentro de la economía colombiana. Una aplicación del modelo se puede ver en Haddad et al. (2009), donde se estudian los efectos regionales que tendría una disminución de los aranceles nacionales.

El objetivo de este estudio es medir el encadenamiento de los sectores económicos desde una perspectiva regional. Conocer los sectores que mayores encadenamientos generan es un insumo importante para el diseño de políticas 
económicas, sobre todo en momentos de recesión. Los incentivos productivos tendrán una mayor difusión en las economías regionales si se aplican a las actividades económicas que mejor se conecten con el resto de sectores, ya que logran 'arrastrar' o 'impulsar' la producción en momentos críticos. Los sectores líderes son aquellos que se encadenan tanto hacia adelante como hacia atrás, por lo que su identificación es clave para diseñar incentivos económicos. En este sentido, estudiar ambos tipos de encadenamiento es muy importante, y el diseño de una política económica eficiente debe tener en cuenta la existencia de los dos.

El resto del trabajo se divide en seis secciones. A continuación, se hace una revisión de la literatura sobre las aplicaciones empíricas del modelo insumo producto. En la sección 2 se describe la metodología usando un énfasis regional, mientras que la sección 3 expone el concepto de encadenamiento y los indicadores que se utilizan para medirlo. La sección 4 da cuenta brevemente de los datos y las distintas fuentes utilizadas. La sección 5 ilustra los resultados del estudio y la última finaliza con unas conclusiones.

\section{Revisión de literatura}

Una referencia fundamental para el desarrollo de este trabajo fue el libro de Miller y Blair (2009). En este se encuentra detallada toda la teoría del análisis de insumo producto; por ejemplo, la estimación de las matrices y el cálculo e interpretación de los indicadores de encadenamiento. En particular, para este estudio se utilizó la metodología nacional y regional que se desarrolla en los capítulos 2 y 3 del libro, así como el análisis de multiplicadores del capítulo 6 y la metodología básica de los encadenamientos del capítulo 12.

Para el caso colombiano existen algunos estudios nacionales que utilizan el enfoque de insumo producto. Hernández (2012) hace una estimación de la matriz nacional utilizando la información publicada por el DANE, la cual cuenta con dos matrices para el año 2007: la de utilización y la de oferta. Esta división nace entre otras razones debido a la existencia de producción secundaria, pues hay sectores en la economía que producen simultáneamente varios bienes o servicios, sin que necesariamente sean el objetivo principal de su actividad. La matriz de utilización contiene la información sobre el uso de los insumos por parte de los sectores productivos. En otras palabras, cuánto de cada bien utilizó cada sector para desarrollar su actividad económica. Y la matriz de oferta contiene cuánto de cada bien fue producido por cada sector.

A partir de la matriz insumo producto estimada, Hernández (2012) analiza los eslabonamientos hacia adelante y hacia atrás de los sectores productivos. De esta forma, identifica los sectores que más encadenamientos generan y los 
clasifica de acuerdo con su conexión con el resto de la economía. Encuentra que los sectores asociados al refinamiento de petróleo y a la producción de químicos y plásticos, entre otros, son los de mayores impactos hacia adelante y hacia atrás.

Por otro lado, Bonet (2000) presenta una estimación de un modelo insumo producto para el Caribe colombiano, donde se analizan eslabonamientos de ingreso, producción y empleo. Esto se lleva a cabo resolviendo un sistema de ecuaciones que caracterizan el comportamiento de 25 sectores económicos en la región. En este estudio las relaciones intersectoriales se modelan econométricamente mediante las primeras derivadas, cuya solución debió ser hallada de manera numérica en algunos casos. Se concluye que el diseño de una política de estímulo económico depende del tipo de fomento que se busca: producción, ingreso o empleo. Para el primero, el sector que brindaba los mayores beneficios en la región era el de agricultura, en el segundo fue el de servicios y en el tercero era el sector de las manufacturas.

Otro estudio regional es el de Lozano et al. (2006). En este se realiza la estimación de la matriz insumo producto para el Valle del Cauca utilizando el método RAs biproporcional, para el cual se emplean tanto datos nacionales del DANE como información regional. Clasifican la economía del Valle en 42 sectores, de los cuales se identifican los más importantes según el encadenamiento que presenten ante el resto de la economía. Esto lo llevan a cabo mediante el cálculo de los coeficientes de Rasmussen, que son los eslabonamientos totales (directos e indirectos), hacia adelante y hacia atrás, de cada sector. Se clasifican los sectores de la economía en sectores clave (presentan ambos), impulsores (hacia atrás), estratégicos (hacia adelante) o independientes (ninguno de los dos).

Los estudios regionales son muy importantes, ya que ayudan a identificar comportamientos y patrones sectoriales que son locales. Sin embargo, debido a que son estudios independientes, no permiten hacer comparaciones entre las distintas regiones porque las metodologías utilizadas y los períodos de estudio suelen diferir. El trabajo de Cordi (1999) hace un avance en este sentido, puesto que realiza la estimación de matrices de contabilidad social ${ }^{1}$ para ocho regiones del país. Esto les permite analizar de manera comparativa la situación de las distintas regiones en temas como producción, generación y distribución de ingresos factoriales, ahorro y financiamiento externo. El estudio muestra que Bogotá mantiene una posición predominante frente a las otras regiones del país, principalmente en la producción, el monto de deman-

1 Según Hernández (2003), “una matriz de contabilidad social contiene la información provista por el Sistema de Cuentas Nacionales, detallando los vínculos entre la oferta y la demanda de productos en la economía y las cuentas de los sectores institucionales". 
da final, las importaciones del resto del mundo, los niveles y tasas de ahorro, y los pagos factoriales. También muestra que Bogotá, Antioquia y Valle del Cauca tienen economías productivas enfocadas hacia el sector secundario y terciario, mientras que las regiones Pacífico y Orinoquia-Amazonia dependen en gran medida de sus sectores primarios.

Bonet (2006) analiza la dependencia inter e intrarregional en siete regiones de Colombia utilizando un modelo insumo producto multirregional estimado para los años 1985, 1992 y 1997. El trabajo encuentra una interdependencia espacial limitada y un cambio estructural que se manifiesta en el traslado de los sectores clave desde los sectores primarios y secundarios hacia el terciario, evolución que es frecuentemente observada en el proceso de desarrollo económico. En la medida en que los sectores con los más fuertes eslabonamientos se encuentran concentrados en las regiones prósperas, existe una alta probabilidad de que las desigualdades regionales existentes permanezcan en el mediano plazo. ${ }^{2}$

Otro estudio que analiza regionalmente los encadenamientos productivos es el de Perobelli et al. (2010). Los autores evalúan el grado de dependencia que tiene la economía nacional frente a sus departamentos más importantes; es decir, busca encontrar cuáles son los que generan el mayor impacto sobre el resto de la economía, por el grado de sus encadenamientos hacia adelante y hacia atrás. Para esto utilizan el método de extracción, que se basa en 'eliminar' un departamento de la economía y observar el impacto que esto genera sobre el resto de los departamentos. Midiendo ambos efectos, encuentran que la región central del país concentra los impactos más grandes sobre la economía nacional: Bogotá, Valle del Cauca, Antioquia, Santander y Cundinamarca. El único departamento ubicado por fuera de esta zona que genera un impacto similar es Atlántico. Concluyen que existe un patrón económico de centro-periferia, donde los departamentos ubicados en la periferia del país participan con efectos de encadenamiento débiles.

\section{Análisis de insumo producto regional}

En una economía es posible clasificar los bienes y servicios que circulan de acuerdo con el sector que los produce. Los productos del café, la pesca y la ganadería se agrupan dentro del sector agropecuario, mientras que la extrac-

2 Galvis y Hahn (2015) encuentran que entre 1993 y 2012 las disparidades económicas regionales no se han reducido, principalmente por los efectos espaciales que se generan por la presencia de spillovers en la acumulación del capital humano y físico. 
ción de recursos naturales como el carbón o el petróleo se clasifican como sector minero. Sin embargo, debido a la existencia de producción secundaria o byproducts, suele suceder que un sector $i$ de la economía produzca bienes de tipo $j$. Este es el caso sobre todo para enfoques sectoriales muy desagregados.

El análisis de insumo producto considera que todos los bienes y servicios en una economía pueden tener uno de dos fines. Por un lado, pueden ser usados como insumos en la producción de otros bienes y servicios. En este caso, a la porción del bien $i$ que el sector $j$ demanda para producir sus bienes y servicios se le conoce como demanda intermedia, y en la notación de insumo producto se representa con la variable $z_{i j}$. Sin embargo, el bien $i$ también puede ser utilizado para satisfacer demanda final, que es donde se clasifican el consumo de los hogares y las exportaciones de la economía, entre otros rubros. Esta se clasifica con la variable $f_{i}$. De esta manera, la distribución del bien $i$ dentro de la economía, entre un número $n$ de sectores, se puede ver de la siguiente forma ${ }^{3}$ :

$$
x_{i}=z_{i 1}+z_{i 2}+\ldots+z_{\text {in }}+f_{i}=\sum_{j=1}^{n} z_{i j}+f_{i}
$$

Donde $x_{i}$ representa la producción del sector $i, z_{i j}$ representa la demanda intermedia del bien $i$ realizada por el sector $j, y f_{i}$ es la demanda final del bien $i$. La proporción de la demanda intermedia del bien $i$ hecha por el sector $j$, sobre el total del bien $j$ producido en la economía, se define como el coeficiente técnico:

$$
a_{i j}=\frac{x_{i j}}{x_{j}}
$$

El coeficiente $a_{i j}$ representa la participación que tiene el insumo $i$ en la producción del bien $j$. La ecuación (2) puede reescribirse para despejar $z_{i j}$ :

$$
z_{i j}=a_{i j} x_{j}
$$

Esta ecuación se utiliza para reemplazar $z_{i j}$ en (1), para cada sector $j$ :

$$
x_{i}=a_{i 1} x_{1}+\ldots+a_{i n} x_{n}+f_{i}=\sum_{j=1}^{n} a_{i j} x_{j}+f_{i}
$$

3 Toda la información de las variables (demanda intermedia, demanda final, producción) se encuentra en valores monetarios. 
Teniendo en cuenta que existe un número $n$ de sectores productivos, existirá entonces un número igual de ecuaciones que describa la distribución de todos los sectores (la ecuación anterior para cada sector $i=1,2, \ldots, n$ ). Las $n$ ecuaciones que surgen para los sectores de la economía pueden ser organizadas en un sistema matricial de la siguiente manera:

$$
x=A x+f
$$

Donde las matrices $x, A$ y $f$ se definen así:

$$
x=\left[\begin{array}{c}
x_{1} \\
\vdots \\
x_{n}
\end{array}\right] A=\left[\begin{array}{ccc}
a_{11} & \cdots & a_{1 n} \\
\vdots & \ddots & \vdots \\
a_{n 1} & \cdots & a_{n n}
\end{array}\right] f=\left[\begin{array}{c}
f_{1} \\
\vdots \\
f_{n}
\end{array}\right]
$$

La matriz $A$ es la matriz que contiene los coeficientes técnicos definidos en (2). Al multiplicar las matrices $A$ y $x$ se obtienen las demandas intermedias de los bienes para cada uno de los sectores; es decir, $z_{i j}$. Por lo tanto, (5) es la versión matricial de la ecuación (1). Si se despeja la matriz $x$ de la ecuación (5), se obtiene la ecuación básica de análisis utilizada por la teoría del insumo producto:

$$
\begin{gathered}
x=L f \\
L=(I-A)^{-1}
\end{gathered}
$$

La matriz $L$ se conoce como la inversa de Leontief o la matriz de requerimientos totales. Los elementos que la componen muestran la variación en la producción de la economía que se genera al cambiar el vector de demanda final. Es decir: si la demanda final pasa de $f$ a $f^{\prime}$, la nueva matriz de producción económica que satisface esa nueva demanda estaría dada por $x^{\prime}=L f^{\prime}$. Estos cambios en la demanda final se originan en eventos que pueden ser desde la implementación de una política fiscal expansiva (por ejemplo, un aumento en el gasto del gobierno), hasta choques externos sobre las exportaciones (por ejemplo, un nuevo tratado de libre comercio - TLC-).

Los elementos $l_{i j}$ de la matriz de Leontief se interpretan como el efecto de la demanda final del bien $j$ sobre la producción del bien $i$. Este efecto se da porque, cuando se demanda una mayor cantidad de $j$, el sector compra más insumos necesarios para su producción, entre los cuales se puede encontrar el 
bien $i$. De esta forma, la demanda final de $j$ afecta la producción de $i$. Por ejemplo, si $l_{i j}$ tiene un valor de 0,5 , significa que un incremento de 1 en la demanda final de $j$ aumenta la producción de $i$ en 0,5 , medido en unidades monetarias.

Hasta este punto, la teoría del análisis insumo producto permite medir la importancia de los distintos sectores dentro de la economía nacional. Sin embargo, es posible pensar que la economía se encuentra desagregada no solo en sectores, sino también en regiones. Este es el caso que estudia el análisis de insumo producto multirregional, donde cada región genera su propia producción de bienes y servicios que, a su vez, puede ser distribuida tanto en esa región como en otras regiones. Para el caso de una economía que tiene dos regiones ( $U$ y $V$ ) y dos sectores ( 1 y 2 ), asumiendo que las regiones tienen los mismos sectores, la producción del sector 1 en la región $U$ se distribuye así como en la ecuación (1):

$$
x_{1}^{u}=x_{11}^{u u}+x_{12}^{u u}+x_{1 a}^{u v}+x_{12}^{u v}+f_{1}^{u}
$$

La notación cambia con respecto a la usada para una matriz nacional, debido a que va a ser necesario identificar no solo los flujos de bienes entre los sectores, sino también entre las distintas regiones. Un sector cualquiera localizado en cierta región puede utilizar insumos tanto de sectores que se encuentran en su misma región como de los que están en otras regiones. Cuando la demanda intermedia surge de la misma región se denomina un flujo intrarregional, mientras que si proviene de otras regiones es un flujo interregional. Para la ecuación (8), $x_{1}{ }^{u}$ representa la producción de 1 hecha en la región $U ; z_{11}{ }^{u v}$ es la cantidad del bien 1 producido en la región $U$, demandada por el sector 1 en la región $V$ (y así para el resto de flujos); y $f_{1}^{u}$ es la demanda final por el bien 1 de la región $U$.

Al haber dos tipos de flujos (intrarregionales e interregionales), existen también dos tipos de coeficientes: los intrarregionales y los interregionales. Similar a lo establecido en la ecuación (2), cada coeficiente se define de la siguiente manera:

$$
\begin{aligned}
& a_{i j}^{u u}=\frac{z_{i j}^{u u}}{x_{j}^{u}} \\
& a_{i j}^{v u}=\frac{z_{i j}^{v u}}{x_{j}^{u}}
\end{aligned}
$$


El coeficiente intrarregional $a_{i j}{ }^{u u}$ muestra la participación que tiene la demanda del bien $i$ en la región $U$ hecha por el sector $j$ en la región $U\left(z_{i j}{ }^{u l u}\right)$, dentro de la producción del bien $j$ en su misma región $\left(x_{j}^{u}\right)$. De forma similar, el coeficiente interregional $a_{i j}{ }^{v u}$ muestra esta misma proporción pero relacionando los flujos provenientes de los sectores económicos ubicados en las otras regiones $\left(z_{i j}^{v u}\right)$.

Para el caso de una economía con dos regiones, los vectores de demanda final y producción se construyen así:

$$
x=\left[\begin{array}{c}
x_{1}^{u} \\
\vdots \\
x_{n}^{u} \\
x_{1}^{v} \\
\vdots \\
x_{n}^{v}
\end{array}\right] f=\left[\begin{array}{c}
f_{1}^{u} \\
\vdots \\
f_{n}^{u} \\
f_{1}^{v} \\
\vdots \\
f_{n}^{v}
\end{array}\right]
$$

Se puede ver que en los vectores $x$ y $f$ se ordena la información de producción y demanda final para ambas regiones. Finalmente, se define la matriz de coeficientes de la siguiente manera. En la diagonal de la matriz se encuentran unas submatrices de coeficientes intrarregionales para cada región, $A^{u t}$ y $A^{v v}$. Por fuera de la diagonal se ubican las submatrices de coeficientes interregionales, que en este ejemplo únicamente serán dos: la submatriz que muestra las proporciones de los flujos desde la región $U$ hacia la región $V\left(A^{u v}\right)$ y desde la región $V$ hacia la región $U\left(A^{v u}\right)$. Todas estas submatrices de la matriz $A$ son cuadradas de tamaño $n$ y contendrán los coeficientes interregionales e intrarregionales respectivos. A continuación se muestra a modo de ejemplo la composición de $A^{u u}$ y $A^{u v}$ :

$$
A^{u u}=\left[\begin{array}{ccc}
a_{11}^{u u} & \cdots & a_{1 n}^{u u} \\
\vdots & \ddots & \vdots \\
a_{n 1}^{u u} & \cdots & a_{n n}^{u u}
\end{array}\right] A^{u v}=\left[\begin{array}{ccc}
a_{11}^{u v} & \cdots & a_{1 n}^{u v} \\
\vdots & \ddots & \vdots \\
a_{n 1}^{u v} & \cdots & a_{n n}^{u v}
\end{array}\right]
$$

La matriz $A$, que agrupará las cuatro matrices de coeficientes regionales, se verá de la siguiente forma: 


$$
A=\left[\begin{array}{ll}
{\left[A^{u u}\right]} & {\left[A^{u v}\right]} \\
{\left[A^{v u}\right]} & {\left[A^{v v}\right]}
\end{array}\right]
$$

Al construir las matrices de coeficientes, producción y demanda final de la anterior manera, la ecuación (6) de análisis insumo producto para el caso de múltiples regiones se mantiene igual que en el caso de una sola región:

$$
\begin{gathered}
x=L f \\
L=(I-A)^{-1}
\end{gathered}
$$

La estructura de la matriz de Leontief regional es similar a la de la matriz de coeficientes regionales. Esta se divide en submatrices, dependiendo del número de regiones del estudio. Para una economía con $1,2, \ldots R$ regiones, se verá así:

$$
L=\left[\begin{array}{ccc}
{\left[L^{11}\right]} & \cdots & {\left[L^{1 R}\right]} \\
\vdots & \ddots & \vdots \\
{\left[L^{R 1}\right]} & \cdots & {\left[L^{R R}\right]}
\end{array}\right]
$$

Las submatrices que componen la matriz $L$ son cuadradas de tamaño $n$ y contienen los multiplicadores de producto para cada región. Esto significa que la matriz $L^{11}$ estará compuesta por los elementos $l_{i j}{ }^{11}$, los cuales representan el impacto en la producción de $i$ en la región 1, cuando la demanda final del bien $j$ en la región 1 sube en una unidad.

$$
L^{11}=\left[\begin{array}{ccc}
l_{11}^{11} & \cdots & l_{1 n}^{11} \\
\vdots & \ddots & \vdots \\
l_{n 1}^{11} & \cdots & l_{n n}^{11}
\end{array}\right]
$$

\section{Medidas de encadenamiento}

Existen varias formas de ver los encadenamientos entre sectores y regiones. Este estudio utilizará dos metodologías. La primera emplea los elementos de 
la matriz inversa de Leontief, que permiten identificar efectos de naturaleza intrarregional e interregional entre los departamentos. Evaluando el cambio que ha tenido lugar en estos indicadores entre dos años se pueden observar los patrones de encadenamiento tanto nacional como regional. Sin embargo, estos índices no tienen en cuenta el tamaño del sector estudiado. Es deseable conocer si las medidas de encadenamiento se ven afectadas al tener en cuenta el peso del sector. Por esta razón, se utiliza una segunda metodología, que es la del encadenamiento puro. Estos indicadores incorporan la producción total del sector dentro del cálculo del indicador. No obstante, no permite identificar patrones intra- o interregionales, ya que se define únicamente para medir los efectos sectoriales dentro de las economías locales.

\subsection{Matriz inversa de Leontief}

La primera medida de encadenamiento regional se construirá utilizando la información de los elementos en la matriz inversa de Leontief. Las submatrices que se encuentran sobre la diagonal de $L$ muestran la relación entre los sectores dentro de la misma región. En este sentido, para observar el grado de dependencia económica que presenta cada una de las regiones a nivel local, se utilizará una medida que consiste en la suma de todos los encadenamientos intrarregionales. En otras palabras, la suma de los elementos de cada submatriz sobre la diagonal de $L$. Con estos indicadores se busca identificar a los departamentos que más dependen económicamente de sus propios sectores productivos.

De esta forma se definen los indicadores intrarregionales, para cada año:

$$
\operatorname{Intra}{ }^{R}=\sum_{i} \sum_{j} l_{i j}^{R R}
$$

Para medir los encadenamientos interregionales se realiza el mismo procedimiento. Sin embargo, y a diferencia de los indicadores intrarregionales, en este caso existen dos tipos distintos de encadenamientos. El primero es el impacto que genera una región por su oferta de bienes y servicios al resto de regiones. Este se denominará el encadenamiento interregional hacia adelante. Y el segundo es el impacto generado por la región al demandar bienes y servicios desde el resto de regiones, que son usados como insumos en su proceso de producción. Este se denominará el encadenamiento interregional hacia atrás. Los indicadores de encadenamiento intrarregionales construidos no tienen estas dos versiones, dado que la región de origen y la región de destino son la misma. 
Los indicadores interregionales se definen de la siguiente manera:

$$
\text { (atrás) Intra }=\sum_{Q} \sum_{i} \sum_{j} l_{i j}^{Q R}
$$

Los indicadores intra- e interregionales utilizados en este trabajo se pueden entender más fácil de manera gráfica en la figura 1, donde se muestra una matriz de Leontief para tres regiones $(A, B$ y $C)$ y tres sectores $(1,2$ y 3$)$.

\begin{tabular}{|c|c|c|c|c|c|c|c|c|}
\hline A1 & A2 & A3 & B1 & B2 & B3 & $\mathrm{C} 1$ & C2 & C3 \\
\hline $\mathrm{A} 1$ & $x$ & $X$ & - & - & - & - & - & - \\
\hline A2 & $x$ & $x$ & - & - & - & - & - & - \\
\hline A3 & $x$ & $X$ & - & - & - & - & - & - \\
\hline B1 & $\mathrm{O}$ & $\mathrm{O}$ & & & & & & \\
\hline $\mathrm{O}$ & $\mathrm{O}$ & $\mathrm{O}$ & & & & & & \\
\hline $\mathrm{O}$ & $\mathrm{O}$ & $\mathrm{O}$ & & & & & & \\
\hline $\mathrm{C} 1$ & $\mathrm{O}$ & $\mathrm{O}$ & & & & & & \\
\hline C2 & $\mathrm{O}$ & $\mathrm{O}$ & & & & & & \\
\hline $\mathrm{O}$ & $\mathrm{O}$ & $\mathrm{O}$ & & & & & & \\
\hline
\end{tabular}

Figura 1. Construcción de indicadores intra- e interregionales: matriz inversa de Leontief

Fuente: elaboración del autor.

Para la región $A$, la suma de los elementos $X$ es el indicador de encadenamiento intrarregional, mientras los elementos - y $O$ representan los encadenamientos interregionales. El indicador hacia adelante es la suma de los elementos -, mientras que el indicador con el encadenamiento hacia atrás es la suma de los elementos $O$. De esta misma forma se pueden calcular para las otras regiones, lo que permite identificar cuáles departamentos generan unos mayores encadenamientos en su propia región y en el resto del país.

Las medidas de encadenamiento se estandarizarán de acuerdo con el promedio departamental observado para cada período. Esto significa que los resultados presentados muestran la relación del encadenamiento departamental frente al promedio de todos los departamentos. De esta forma, si un departamento en particular muestra un indicador de 1,05, quiere decir que su encadenamiento para ese año se encuentra un $5 \%$ por encima del promedio departamental. Una vez estandarizados, se ordenarán los departamentos 
según el valor de sus indicadores para cada año. Esto permitirá conocer los departamentos cuyas dependencias económicas (intra- o interregionales) cambiaron en relación con el resto de regiones.

Por último, se calculan los indicadores de encadenamiento de cada sector para el agregado nacional. Estos son la suma por filas (para los encadenamientos hacia adelante) y por columna (para los encadenamientos hacia atrás) de los elementos de la matriz $L$, para uno de los siete sectores identificados. Los indicadores también se estandarizan usando el promedio sectorial observado.

\subsection{Encadenamiento puro}

Los indicadores de encadenamiento calculados anteriormente a partir de la matriz inversa de Leontief permiten observar patrones regionales. Sin embargo, estos no tienen en cuenta el tamaño de cada sector. Sonis et al. (1995) proponen la estimación del encadenamiento puro, donde se tiene en cuenta la producción del sector respectivo como insumo en el cálculo de los indicadores. De esta forma, es posible identificar los sectores clave de cada departamento según su grado de encadenamiento, controlando por el tamaño del sector. Así como los encadenamientos regionales obtenidos de la matriz inversa de Leontief, el encadenamiento puro identifica encadenamientos hacia adelante y hacia atrás. Un sector clave en cierto departamento será aquel que tenga un alto grado de impacto sobre su economía regional, tanto por su demanda como por su oferta de insumos. Por sus siglas en inglés, ambos impactos se conocen como PBL (pure backward linkage) y PFL (pure forward linkage), respectivamente.

La medida de encadenamiento puro tiene varias características. En primer lugar, analiza estos impactos sin tener en cuenta el efecto de 'retroalimentación' generado por la dependencia entre sectores. De esta forma, el PBL no tiene en cuenta la demanda intermedia que genera la economía por ese sector, mientras que el PFL no tiene en cuenta la oferta de insumos de ese sector hacia la economía. En otras palabras, es como si cada sector se 'desconectara' de la economía local y se calculara el grado de encadenamiento que este genera, olvidando los efectos de retroalimentación. Por otro lado, estos indicadores solo están construidos para medir los impactos en las economías locales. Esto implica que no es posible ver medidas de encadenamiento puro interregionales.

Para calcular los índices de encadenamiento puro, es necesario tener las matrices de coeficientes intrarregionales, nombradas anteriormente como $A^{u u}$. Esta matriz se puede descomponer en cuatro partes, para un sector $j$ determinado: 


$$
A^{u u}=\left[\begin{array}{cc}
A_{j j} & A_{j r} \\
A_{r j} & A_{r r}
\end{array}\right]
$$

- $A_{j j}$ es el coeficiente de la demanda intermedia hacia el mismo sector $j$.

- $A_{j r}$ es un vector fila de tamaño (n-1), que contiene la información de la oferta de insumos desde el sector $j$ al resto de sectores.

- $A_{r j}$ es un vector columna de tamaño (n-1), que contiene la información de las demandas intermedias hechas por el sector $j$ al resto de sectores.

- $A_{r r}$ es una matriz cuadrada de tamaño (n-1), que contiene los coeficientes del resto de la economía local, excluyendo al sector $j$.

Para construir los indicadores de encadenamiento puro, es necesario también definir los siguientes elementos:

- $i_{r r}^{\prime}$ : un vector fila de unos de tamaño $(n-1)$

- $\Delta_{r}=\left(I-A_{r r}\right)^{-1}$

- $q_{j j}$ : la producción total del sector $j$

- $q_{r r}:$ un vector columna de tamaño $(n-1)$ con la producción del resto de sectores, excluyendo al sector $j$

Cada una de las regiones tiene en consecuencia un total de $n$ descomposiciones de su matriz de coeficientes intrarregionales. Se realiza la estimación del PBL y el PFL de la siguiente manera:

$$
\begin{gathered}
\text { PBL: } i_{r r}^{\prime} \Delta_{r} A_{r j} q_{j j} \\
\text { PFL: } A_{j r} \Delta_{r} q_{r r}
\end{gathered}
$$

Luego de estimados, se estandarizan los indicadores según el valor promedio de cada departamento. Siguiendo la metodología de Bonet (2006), los sectores que tengan impactos hacia adelante o hacia atrás por encima del promedio departamental se muestran como sectores importantes. Aquellos que muestren ambos efectos, se clasificarán como sectores clave.

Por último, se realizarán dos análisis más agregados, cuyo propósito es identificar tendencias en la distribución de los impactos locales. Para ello se calcula la medida de encadenamiento puro total (pure total linkage), que se define como la suma entre ambos indicadores: 


$$
\mathrm{PTL}=\mathrm{PBL}+\mathrm{PFL}
$$

El PTL es una medida que resume el tamaño del impacto de cada sector sobre su economía local. Al agrupar el PBL y el PFL se pueden observar los sectores que mayor impacto generan, independientemente de que sea por sus efectos hacia adelante como por sus efectos hacia atrás. Este cálculo se realizará para los departamentos a través de la suma de los indicadores sectoriales de cada departamento y para los siete sectores mediante la agregación de los indicadores departamentales de cada sector. Luego se estandarizan utilizando el promedio sectorial o departamental respectivo.

\section{Datos}

Para la realización de este trabajo se utilizaron dos conjuntos de información que se configuraron para la economía colombiana de los años 2004 y 2012. Estas bases de datos, construidas bajo el formato de insumo producto, fueron elaboradas en el proceso de estimación y actualización del modelo CEER, un modelo multirregional de equilibrio general computable. El modelo CEER es un trabajo conjunto realizado por el Centro de Estudios Económicos Regionales (CEER) y la Fundación Instituto de Investigación Económica (FIPE) de la Universidad de Sao Paulo en Brasil. La estimación de la matriz para el año 2012 se basó en dos etapas. La primera consistió en la estimación de una matriz insumo producto nacional, usando las matrices de utilización y oferta producidas por el DANE. Para hacer la transformación del enfoque productosector al enfoque sector-sector, se asumió que la tecnología era basada en el sector de producción (industry-based technology). ${ }^{4}$ Las matrices se construyeron a precios básicos, por lo que se debieron sustraer los márgenes generados por los sectores de comercio y transporte. La segunda etapa consistió en la regionalización de la matriz nacional para los 33 departamentos. Esta se llevó a cabo usando el enfoque conocido en la literatura como Chenery-Moses. Tanto la elaboración de la matriz nacional como su posterior regionalización se encuentran descritas en Haddad et al. (2016).

El modelo se configuró en ambos años para 7 sectores y 33 departamentos. La agregación sectorial del modelo es: (1) agropecuario, (2) minería, (3) industria manufacturera, (4) construcción, (5) transporte, (6) administración

4 Para mayor información sobre el enfoque producto-sector (commodity-by-industry approach), ver Miller y Blair (2009), capítulo 5. 
pública y (7) otros servicios. En esta última categoría se encuentran las ramas de generación y distribución de energía eléctrica y gas, comercio, restaurantes y hoteles, telecomunicaciones, intermediación financiera, actividades inmobiliarias, servicios a las empresas, servicios sociales, de educación y salud de mercado, eliminación de desperdicios y actividades de esparcimiento de mercado. La anterior agregación permite identificar y comparar los sectores productivos en ambos momentos del tiempo.

En cuanto a las regiones utilizadas, para el presente trabajo se decidió agrupar a los nuevos departamentos en una sola región. Esto se debe a que son economías muy pequeñas y con escasas relaciones comerciales frente al resto del país, lo que dificulta una estimación precisa de sus encadenamientos interregionales. Si bien esta agregación no permite estudiar las economías de estos departamentos por separado, los encadenamientos para la región agregada obtenidos son más precisos.

\section{Resultados}

\subsection{Matriz inversa de Leontief: indicadores intrarregionales}

La tabla 1 contiene los indicadores intrarregionales. Los departamentos se ordenaron, para cada año, de acuerdo con las magnitudes de sus indicadores.

Tabla 1. Ranking indicadores intrarregionales 2004-2012

\begin{tabular}{lcccc}
\hline \multicolumn{1}{c}{ Departamento } & Indicador 2004 & Ranking 2004 & Indicador 2012 & Ranking 2012 \\
\hline Antioquia & 1,053 & 6 & 1,111 & 1 \\
Atlántico & 1,054 & 5 & 1,035 & 6 \\
Bogotá, D. C. & 1,017 & 10 & 1,059 & 3 \\
Bolívar & 1,004 & 13 & 1,040 & 4 \\
Boyacá & 0,972 & 19 & 1,029 & 7 \\
Caldas & 1,122 & 1 & 0,996 & 13 \\
Caquetá & 0,979 & 16 & 0,936 & 24 \\
Cauca & 1,086 & 2 & 1,010 & 9 \\
Cesar & 0,914 & 23 & 0,962 & 20 \\
Chocó & 0,991 & 15 & 0,920 & 25 \\
Córdoba & 1,022 & 9 & 0,954 & 21 \\
Cundinamarca & 0,941 & 21 & 0,969 & 19 \\
\hline
\end{tabular}




\begin{tabular}{lcccc}
\hline \multicolumn{1}{c}{ Departamento } & Indicador 2004 & Ranking 2004 & Indicador 2012 & Ranking 2012 \\
\hline Huila & 0,930 & 22 & 0,985 & 15 \\
La Guajira & 0,883 & 25 & 0,944 & 22 \\
Magdalena & 1,014 & 11 & 0,984 & 17 \\
Meta & 1,011 & 12 & 1,010 & 10 \\
Nariño & 0,978 & 17 & 1,006 & 11 \\
Norte de Santander & 0,974 & 18 & 0,999 & 12 \\
Nuevos deptos. & 0,883 & 24 & 1,025 & 8 \\
Quindío & 1,028 & 8 & 0,942 & 23 \\
Risaralda & 1,078 & 3 & 0,984 & 16 \\
Santander & 1,032 & 7 & 1,040 & 5 \\
Sucre & 0,999 & 14 & 0,972 & 18 \\
Tolima & 0,956 & 20 & 0,988 & 14 \\
Valle & 1,077 & 4 & 1,102 & 2 \\
\hline
\end{tabular}

Fuente: cálculos del autor con base en los datos del modelo CEER.

Los departamentos cuyas economías dependían en mayor grado de sus sectores productivos locales en 2004 eran Caldas, Cauca, Risaralda y Valle del Cauca, en ese orden; y los que menos dependían eran La Guajira, el conjunto de los nuevos departamentos y el Cesar. Nótese que, mientras los primeros son departamentos con economías levemente industrializadas (Valle del Cauca en mayor medida), los últimos son territorios cuya naturaleza económica es extractiva y que presentan un bajo grado de desarrollo económico.

Para 2012 el ranking presenta varios cambios que es importante destacar. Antioquia, Valle y Bogotá aparecen como los de mayor dependencia local, mientras que Chocó, Caquetá y Quindío son los de menor impacto. Nuevamente, los departamentos con mayor influencia intrarregional son regiones con un grado de industrialización elevado: Antioquia, Valle del Cauca y Bogotá se destacan por tener sectores industriales y de servicios más avanzados que el resto del país. Los departamentos que más subieron en el ranking de impactos intrarregionales fueron los nuevos departamentos y Boyacá, mientras que los que más bajaron fueron Risaralda y Quindío.

Vale la pena traer a discusión el fenómeno estudiado por Okazaki (1989) para la economía japonesa y por Hewings et al. (2010) para la economía de Chicago, conocido como el hollowing out. Se argumenta que el desarrollo de una economía sucede por etapas, donde en cada etapa hay procesos sectoriales y comerciales diferentes. En la primera etapa la economía crece y se desarrolla 
demandando bienes y servicios locales, que son los que tiene a su alcance, por lo que incrementa sus encadenamientos dentro de su misma región. Sin embargo, llega un momento del desarrollo en el que los sectores productivos buscan oferentes más especializados, o economías de escala que produzcan sus insumos de manera más competitiva, por lo que comienzan a sustituir flujos de bienes y servicios de producción local por aquellos producidos en otras regiones, o países.

Es interesante ver que en Colombia, utilizando los indicadores de encadenamientos derivados de la matriz inversa de Leontief, los departamentos más desarrollados son los que presentan mayores encadenamientos locales. Este hallazgo no soporta el fenómeno de hollowing out encontrado en la literatura y, por el contrario, las economías más avanzadas siguen mostrando mayores encadenamientos en el interior de sus aparatos productivos.

\subsection{Matriz inversa de Leontief: indicadores interregionales}

Los indicadores interregionales se calcularon en sus dos versiones, tanto para los encadenamientos hacia adelante como hacia atrás. En la tabla 2 se presenta la posición de cada departamento en el ranking nacional. ${ }^{5}$

Los indicadores interregionales presentan las siguientes características:

1) Los departamentos con economías más desarrolladas muestran indicadores de encadenamiento interregionales altos hacia adelante, pero bajos hacia atrás. Bogotá, Antioquia, Valle del Cauca y Atlántico aparecen en lo más alto del ranking en la parte izquierda de la tabla, mientras que en la parte derecha se encuentran de últimos. Este es un patrón interesante, paralelo al hallado en los indicadores de encadenamiento intrarregionales, donde las economías más industrializadas se encontraban de primeras.

2) Los departamentos con economías intermedias o poco desarrolladas no presentan un patrón tan claro como los anteriores. No obstante, se pueden observar algunos casos particulares como los de Chocó y Caquetá, de bajo desarrollo económico, que muestran bajos encadenamientos hacia adelante y relativamente altos encadenamientos hacia atrás. Quindío, una economía pequeña y en desarrollo, también muestra un patrón similar.

5 Los indicadores se presentan en el anexo 1. 
Tabla 2. Ranking indicadores interregionales 2004-2012

\begin{tabular}{|c|c|c|c|c|}
\hline \multirow{2}{*}{ Departamento } & \multicolumn{2}{|c|}{ Hacia adelante } & \multicolumn{2}{|c|}{ Hacia atrás } \\
\hline & 2004 & 2012 & 2004 & 2012 \\
\hline Antioquia & 3 & 3 & 19 & 25 \\
\hline Atlántico & 4 & 6 & 20 & 21 \\
\hline Bogotá, D. C. & 1 & 1 & 25 & 23 \\
\hline Bolívar & 7 & 5 & 15 & 22 \\
\hline Boyacá & 13 & 10 & 2 & 19 \\
\hline Caldas & 12 & 13 & 18 & 13 \\
\hline Caquetá & 24 & 25 & 3 & 2 \\
\hline Cauca & 16 & 14 & 22 & 17 \\
\hline Cesar & 10 & 19 & 8 & 6 \\
\hline Chocó & 25 & 21 & 1 & 1 \\
\hline Córdoba & 15 & 16 & 7 & 5 \\
\hline Cundinamarca & 6 & 8 & 24 & 7 \\
\hline Huila & 11 & 15 & 10 & 9 \\
\hline La Guajira & 18 & 24 & 23 & 4 \\
\hline Magdalena & 21 & 17 & 11 & 12 \\
\hline Meta & 19 & 7 & 17 & 16 \\
\hline Nariño & 17 & 18 & 13 & 15 \\
\hline Norte de Santander & 20 & 20 & 12 & 14 \\
\hline Nuevos deptos. & 8 & 9 & 14 & 18 \\
\hline Quindío & 23 & 22 & 6 & 3 \\
\hline Risaralda & 14 & 11 & 16 & 10 \\
\hline Santander & 5 & 2 & 5 & 20 \\
\hline Sucre & 22 & 23 & 4 & 8 \\
\hline Tolima & 9 & 12 & 9 & 11 \\
\hline Valle & 2 & 4 & 21 & 24 \\
\hline
\end{tabular}

Fuente: cálculos del autor con base en los datos del modelo CEER.

3) Durante el período de estudio el departamento que más aumentó sus encadenamientos hacia adelante fue el Meta, mientras que los que más los redujeron fueron Cesar y La Guajira (todas economías extractivas). Por otro lado, Cundinamarca y La Guajira fueron los de mayor aumento en sus encadenamientos hacia atrás, mientras que Boyacá y Santander los que mayor disminución presentaron. 
4) Los departamentos con reservas de recursos naturales (en donde se encuentran los de mayor riqueza per capita) muestran encadenamientos relativamente bajos. Esto refuerza la idea de que las economías extractivas, a pesar de generar mucha riqueza, no necesariamente producen mayores oportunidades económicas al resto de los sectores. El Meta, uno de los departamentos con mayor PIB per capita del país por sus reservas de hidrocarburos, muestra indicadores de encadenamiento bajos en términos relativos. Lo mismo sucede con Cesar, La Guajira y los nuevos departamentos, en donde se encuentran Arauca y Casanare.

La tabla 3 expone los indicadores de encadenamiento sectoriales calculados de los elementos de la matriz de Leontief, estandarizados con el promedio sectorial. Los sectores productivos con mayores encadenamientos hacia atrás fueron la industria, la construcción y el transporte. El sector minero tenía un indicador de encadenamiento por encima del promedio para 2004, pero en 2012 presentó una disminución y se ubicó por debajo. El sector agropecuario, la administración pública y los servicios son sectores que no mostraron encadenamientos hacia atrás importantes, lo que significa que demandan pocos insumos del resto de la economía en comparación con los primeros.

Tabla 3. Indicadores sectoriales de la matriz de Leontief

\begin{tabular}{lcccc}
\hline \multirow{2}{*}{ Sector } & \multicolumn{2}{c}{2004} & \multicolumn{2}{c}{2012} \\
\cline { 2 - 5 } & Atrás & Adelante & Atrás & Adelante \\
\hline Agropecuario & 0,83 & 0,87 & 0,89 & 0,87 \\
Minero & 1,09 & 0,67 & 0,82 & 0,83 \\
Industria & 1,26 & 1,75 & 1,17 & 1,45 \\
Construcción & 1,08 & 0,65 & 1,02 & 0,71 \\
Transporte & 1,02 & 0,78 & 1,14 & 0,86 \\
Adm. pública & 0,83 & 0,58 & 0,97 & 0,68 \\
Servicios & 0,89 & 1,70 & 0,97 & 1,61 \\
\hline
\end{tabular}

Fuente: cálculos del autor con base en los datos del modelo CEER.

Los sectores productivos con mayores encadenamientos hacia atrás fueron la industria, la construcción y el transporte. El sector minero tenía un indicador de encadenamiento por encima del promedio para 2004, pero en 2012 presentó una disminución y se ubicó por debajo. El sector agropecuario, la administración pública y los servicios son sectores que no mostraron encadenamientos hacia atrás importantes, lo que significa que demandan pocos insumos del resto de la economía en comparación con los primeros. 
Por otro lado, los sectores de industria y de servicios generan unos encadenamientos hacia adelante elevados. Esto significa que son sectores importantes para la economía nacional por su provisión de bienes y servicios que el resto de sectores usa como insumo en su proceso productivo.

\subsection{Encadenamiento puro}

Los indicadores de encadenamiento puro PFL y PBL fueron estimados y estandarizados utilizando el promedio entre sectores por departamento para cada período. De esta forma, se identificaron los sectores que presentan encadenamientos hacia adelante y hacia atrás. Los sectores líderes son aquellos que muestran ambos. Estos resultados se muestran en la tabla 4.

Los resultados permiten visualizar qué departamentos sufrieron algún cambio en su estructura productiva. Es posible hacer las siguientes observaciones:

1) Los sectores de mayor impacto local son el de industria (3) y el de servicios (7). Estos dos sectores se caracterizan por presentar altos encadenamientos puros tanto hacia adelante como hacia atrás; en la gran mayoría de departamentos del país y para ambos períodos, industria y servicios aparecen como sectores clave. Sin embargo, hay que resaltar que el de servicios redujo en algunos departamentos su importancia.

2) El sector agropecuario (1) y el de transporte (5) redujeron sus impactos en algunos departamentos, mientras que la construcción (4) y la administración pública (6) incrementaron sus encadenamientos puros hacia atrás en gran parte del territorio nacional, lo que refleja el mayor dinamismo de estos dos sectores en los últimos años. Ambos se caracterizan por tener impactos hacia atrás muy importantes.

Tabla 4. Sectores con impactos hacia adelante, hacia atrás y clave por departamento

\begin{tabular}{lcccccc}
\hline \multirow{2}{*}{ Departamento } & \multicolumn{5}{c}{20042} \\
\cline { 2 - 7 } & Adelante & Atrás & Clave & Adelante & Atrás & Clave \\
\hline Antioquia & $1,3,7$ & $3,4,7$ & 3,7 & 3,7 & $3,4,7$ & 3,7 \\
Atlántico & $3,5,7$ & 3,7 & 3,7 & $3,5,7$ & $3,4,5,6,7$ & $3,5,7$ \\
Bogotá, D. C. & 5,7 & 3,7 & 7 & 3,7 & $3,4,6,7$ & 3,7 \\
Bolívar & 3,7 & 3,7 & 3,7 & 3,7 & 3,4 & 3 \\
Boyacá & $1,5,7$ & 3,7 & 7 & 3,7 & 3 & 3 \\
Caldas & $3,5,7$ & $3,5,7$ & $3,5,7$ & 3,7 & $3,4,6,7$ & 3,7 \\
Caquetá & 3,7 & $1,3,6$ & 3 & 7 & 4,6 & \\
& & & & & & Continúa
\end{tabular}




\begin{tabular}{|c|c|c|c|c|c|c|}
\hline \multirow{2}{*}{ Departamento } & \multicolumn{3}{|c|}{2004} & \multicolumn{3}{|c|}{2012} \\
\hline & Adelante & Atrás & Clave & Adelante & Atrás & Clave \\
\hline Cauca & $1,3,7$ & 3 & 3 & 3,7 & $3,4,6$ & 3 \\
\hline Cesar & 5,7 & 2,3 & & 5,7 & 2,6 & \\
\hline Chocó & 5,7 & $2,4,6,7$ & 7 & 7 & 2,6 & \\
\hline Córdoba & $3,5,7$ & $2,3,7$ & 3,7 & 5,7 & 6,7 & 7 \\
\hline Cundinamarca & 5,7 & 3 & & 3,7 & 3,6 & 3 \\
\hline Huila & 5,7 & $2,3,6,7$ & 7 & $3,5,7$ & 4,6 & \\
\hline La Guajira & 5,7 & 2,7 & 7 & 5,7 & 2,6 & \\
\hline Magdalena & $3,5,7$ & $1,3,5,6,7$ & $3,5,7$ & 5,7 & $3,4,6,7$ & 7 \\
\hline Meta & $3,5,7$ & $1,2,3$ & 3 & $3,5,7$ & 2,4 & \\
\hline Nariño & 5,7 & $1,3,4,6,7$ & 7 & 3,7 & $3,6,7$ & 3,7 \\
\hline Norte de Santander & 5,7 & $3,5,6,7$ & 5,7 & $3,5,7$ & $3,6,7$ & 3,7 \\
\hline Nuevos deptos. & 5,7 & 2 & & $3,5,7$ & 2,6 & \\
\hline Quindío & 5,7 & $3,5,7$ & 5,7 & 7 & $3,4,6,7$ & 7 \\
\hline Risaralda & $3,5,7$ & $3,4,5,7$ & $3,5,7$ & $3,5,7$ & $3,6,7$ & 3,7 \\
\hline Santander & $1,5,7$ & 3 & & 3,7 & 3,4 & 3 \\
\hline Sucre & 5,7 & $1,3,6,7$ & 7 & 3,7 & 3,6 & 3 \\
\hline Tolima & $1,5,7$ & 3,7 & 7 & $3,5,7$ & $3,6,7$ & 3,7 \\
\hline Valle & $3,5,7$ & 3,7 & 3,7 & 3,7 & $3,6,7$ & 3,7 \\
\hline
\end{tabular}

Fuente: cálculos del autor con base en los datos del modelo CEER.

3) La minería (2) muestra impactos locales hacia atrás altos en departamentos con disponibilidad de recursos, como, por ejemplo, Cesar, Chocó y Meta.

4) El transporte (5) presenta unos impactos hacia adelante importantes en muchos departamentos. Esto sucedió para ambos años, aunque en 2012 desapareció en algunos departamentos.

Por último, se realizan los análisis agregados por sectores y departamentos, utilizando la medida de encadenamiento puro estandarizada. Estos se muestran en las figuras 2 y 3 . De ambas figuras es posible hacer las siguientes observaciones: 


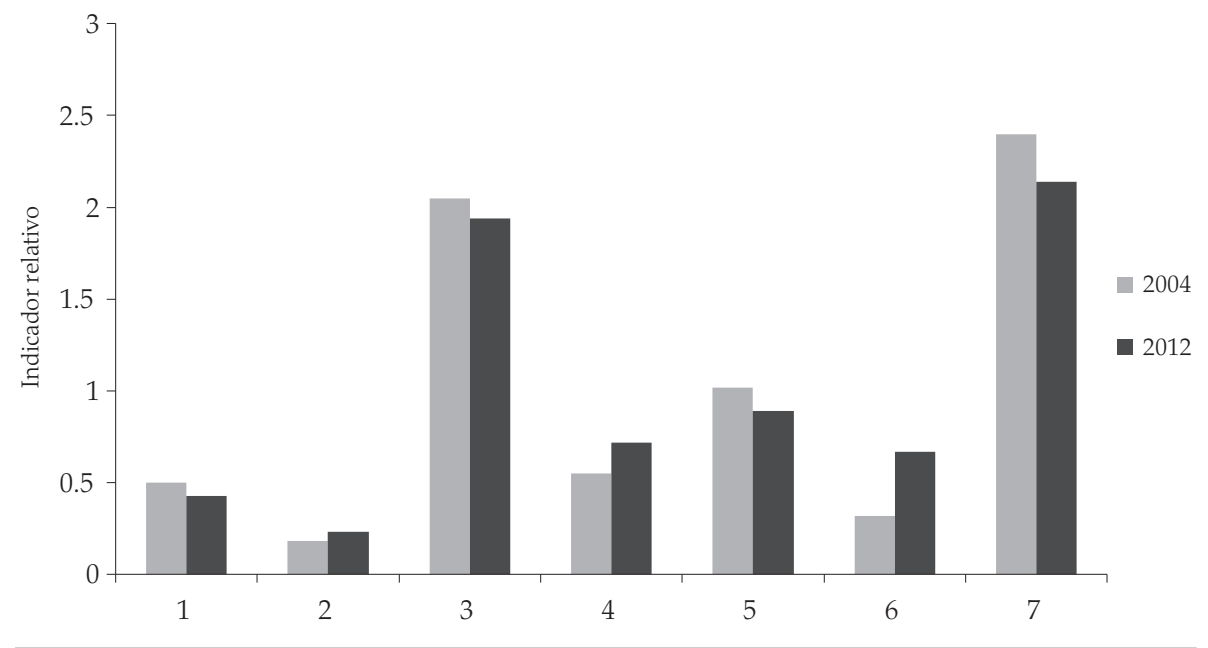

Figura 2. Indicadores de encadenamiento puro por sector, 2004 y 2012

Fuente: cálculos del autor con base en los datos del modelo CEER.

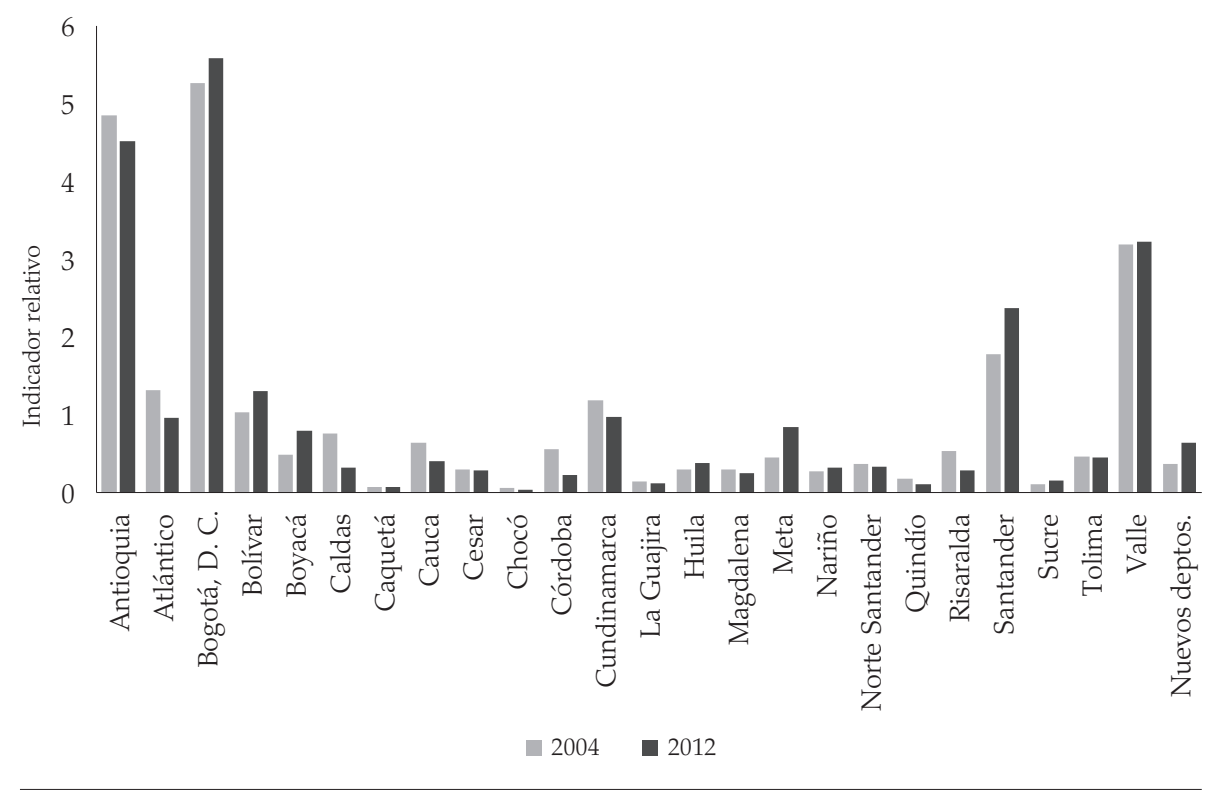

Figura 3. Indicadores de encadenamiento puro por departamento, 2004 y 2012 Fuente: cálculos del autor con base en los datos del modelo CEER.

1) Los sectores productivos que mayores medidas de encadenamiento puro total presentan son la industria y los servicios. Ambos sectores tienen indicadores de casi dos veces el promedio nacional. El resto se encuentra 
por debajo del promedio, donde el que menos muestra es la minería. Entre ambos años aumentó sustancialmente el encadenamiento puro de la administración pública y se redujo el de servicios.

2) Bogotá, Antioquia y Valle del Cauca tienen impactos locales más de tres veces por encima del promedio departamental. Santander, Atlántico, Bolívar y Cundinamarca se encuentran sobre el promedio. El resto de regiones muestra impactos por debajo. El hecho de que sean las tres economías más grandes del país las que generen la mayor cantidad de encadenamiento puro no es una coincidencia. Este indicador incorpora el tamaño de los sectores productivos, por lo que se espera que las economías más grandes generen los mayores impactos. A pesar de esto hay que destacar que el panorama departamental resalta por la poca uniformidad que presenta, y que durante el período de estudio no se presentó un cambio significativo en la estructura regional.

\section{Conclusiones}

En este estudio se analizaron distintas medidas de encadenamientos sectoriales desde una perspectiva regional. Uno de los hallazgos más interesantes es la relación que presentan los departamentos colombianos entre su desarrollo económico y las medidas de encadenamiento derivadas de la matriz inversa de Leontief. La evidencia muestra que en Colombia los departamentos más desarrollados tienen altos encadenamientos intrarregionales e interregionales hacia adelante, pero bajos encadenamientos interregionales hacia atrás. Que las regiones más desarrolladas sean las de mayores encadenamientos intrarregionales es un fenómeno que difiere al observado en estudios como el de Okazaki (1989) y Hewings et al. (2010). Esta diferencia puede deberse a dos razones. La primera es que los departamentos más avanzados en Colombia se encuentran todavía en una etapa del desarrollo temprana, donde se expanden mediante insumos comprados dentro de sus propios territorios, en vez de usar los beneficios que traen las economías de escala y los mercados más competitivos ubicados en otras regiones. La segunda hipótesis es que el alto costo del comercio entre regiones hace que los departamentos colombianos crezcan orientando sus estructuras productivas hacia adentro, debido a que el acceso a mercados externos más competitivos es costoso.

Desde una perspectiva sectorial, se ve que los sectores de mayor encadenamiento en Colombia son la industria y los servicios. Esto es fundamental para el diseño de políticas económicas. La construcción presenta altos encadenamientos hacia atrás, por lo que es un sector importante, ya que impulsa al resto 
mediante su compra de insumos. Sin embargo, al tener en cuenta el tamaño del sector, la industria y los servicios son los de mayor peso en el agregado nacional. Por otro lado, si bien la construcción reporta altos encadenamientos hacia atrás, hacia adelante son bajos. Los servicios son mucho más importantes para la economía por su provisión de insumos, y esto se debe considerar al diseñar políticas económicas. Los sectores productivos generan efectos diferenciados sobre la economía, unos mediante su oferta y otros mediante su demanda. Una política eficiente debe 'echar mano' de ambos, cuando el objetivo sea incentivar la economía en un período de recesión.

Las economías departamentales presentan resultados que algunas veces difieren de lo observado en el agregado nacional. Este es el caso del sector minero. Presenta bajos índices de encadenamiento si se mira la economía del país como un todo; sin embargo, en las regiones con abundancia de este recurso se observa que es un sector importante por sus encadenamientos hacia atrás. Lo mismo sucede con el sector agropecuario. Estas diferencias entre el panorama nacional y las economías regionales deben ser tenidas en cuenta al momento de diseñar políticas públicas cuyo enfoque sea de impacto regional.

Futuros estudios sobre las características productivas de las regiones colombianas deben realizarse con una agregación sectorial más detallada. Al usar solo siete sectores, el presente trabajo ignora muchos detalles que pueden darse en el interior de cada uno de ellos. En particular, la industria y los servicios se caracterizan por contener una gran variedad de actividades productivas que presentan grandes diferencias, como, por ejemplo, el refinamiento de petróleo y la industria textil en el caso del sector industrial. El estudio de estas dinámicas sectoriales se vuelve aún más interesante cuando se enfoca desde un punto de vista regional.

\section{Referencias}

Banguero, H., Duque, H., Garizado, P. A., \& Parra, D. (2006). Estimación de la matriz insumo producto simétrica para el Valle del Cauca - año 1994. Documento presentado en la II Jornada Española de Análisis Input-Output. Zaragoza, España. Recuperado de http:/ / www.unizar.es/jornadasiozaragoza/archivos/pdf/Ponencia_Banguero_Harold.pdf

Bonet, J. (2000). La matriz insumo-producto del Caribe colombiano. Documento de Trabajo sobre Economía Regional, No 15. Cartagena: Banco de la República.

Bonet, J. (junio, 2006). Cambio estructural regional en Colombia: una aproximación con matrices insumo-producto. Revista de Coyuntura Económica. 
Cordi, A. (1999). ¿Se cumplen las verdades nacionales a nivel regional? Archivos de Macroeconomía, N ${ }^{0}$ 121. Departamento de Planeación Nacional.

Dixon, P., \& Rimmer, M. (2004). Disaggregation of results from a detailed general equilibrium model of the us to the state level. Documento de trabajo. Victoria, Australia: Centre of Policy Studies, Monash University. Recuperado de https:/ / www.gtap.agecon.purdue.edu/resources/download/1772.pdf

Haddad, E. A., Bonet J., Hewings G. J., \& Perobelli, F. S. (2009). Spatial aspects of trade liberalization in Colombia: a general equilibrium approach. $\mathrm{Pa}$ pers in Regional Science, 88(4), 699-732.

Haddad, E. A., Faria, W. R., Galvis, L., \& Hahn, L. (2016). Interregional inputoutput matrix for Colombia, 2012. Borradores de Economía, Banco de la República. Recuperado de http:/ / www.banrep.gov.co/sites/default/ files/publicaciones/archivos/be_923.pdf

Hernández, G. (2003). Construcción de una matriz de contabilidad social financiera para Colombia. Documento de trabajo No 223, Archivos de Economía, Departamento Nacional de Planeación.

Hernández, G. (2012). Matrices insumo-producto y análisis de multiplicadores: una aplicación para Colombia. Revista de Economía Institucional, 14(26), 203-221.

Hewings, G. J., Sonis, M., Guo, J., Israilevich, P., \& Schindler, G. (1998). The hollowing-out process in the Chicago economy, 1975-2011. Geographical Analysis, 30(3), 217-233.

Miller, R. E., \& Blair, P. D. (2009). Input-output analysis: foundations and extensions ( $2^{\mathrm{a}}$ ed.). Cambridge University Press.

Okazaki, F. (1989). The hollowing out phenomenon in economic development. Documento presentado en la XI Conferencia Regional de Ciencias del Pacífico, Singapur.

Perobelli, F., Haddad, E., Bonet J., \& Hewings, G. J. (2010). Structural interdependence among Colombian departments. Economic Systems Research, 22(3), 279-300.

Sonis, M., Guilhoto, J., Hewings, G. J., \& Martins, E. B. (1995). Linkages, key sectors, and structural change: some new perspectives. The Developing Economies, 33(3), 243-246. 


\section{Anexos}

\section{Anexo 1. Indicadores interregionales hacia adelante y hacia atrás}

\begin{tabular}{|c|c|c|c|c|}
\hline \multirow{2}{*}{ Departamento } & \multicolumn{2}{|c|}{ Hacia adelante } & \multicolumn{2}{|c|}{ Hacia atrás } \\
\hline & 2004 & 2012 & 2004 & 2012 \\
\hline Antioquia & 3,481 & 2,968 & 0,906 & 0,584 \\
\hline Atlántico & 1,456 & 1,378 & 0,848 & 0,842 \\
\hline Bogotá, D. C. & 6,329 & 4,757 & 0,289 & 0,795 \\
\hline Bolívar & 1,080 & 1,484 & 0,967 & 0,833 \\
\hline Boyacá & 0,479 & 0,645 & 1,424 & 0,900 \\
\hline Caldas & 0,510 & 0,471 & 0,939 & 1,021 \\
\hline Caquetá & 0,097 & 0,066 & 1,326 & 1,267 \\
\hline Cauca & 0,356 & 0,461 & 0,801 & 0,958 \\
\hline Cesar & 0,562 & 0,281 & 1,077 & 1,142 \\
\hline Chocó & 0,056 & 0,256 & 1,551 & 1,285 \\
\hline Córdoba & 0,458 & 0,336 & 1,141 & 1,157 \\
\hline Cundinamarca & 1,141 & 0,939 & 0,312 & 1,126 \\
\hline Huila & 0,518 & 0,419 & 1,063 & 1,066 \\
\hline La Guajira & 0,312 & 0,110 & 0,784 & 1,192 \\
\hline Magdalena & 0,224 & 0,326 & 1,044 & 1,041 \\
\hline Meta & 0,309 & 1,010 & 0,943 & 0,974 \\
\hline Nariño & 0,325 & 0,320 & 1,016 & 0,982 \\
\hline $\begin{array}{l}\text { Norte de San- } \\
\text { tander }\end{array}$ & 0,238 & 0,259 & 1,027 & 1,003 \\
\hline Nuevos deptos. & 0,707 & 0,914 & 1,010 & 0,929 \\
\hline Quindío & 0,147 & 0,221 & 1,204 & 1,217 \\
\hline Risaralda & 0,466 & 0,593 & 0,965 & 1,064 \\
\hline Santander & 1,286 & 3,132 & 1,205 & 0,867 \\
\hline Sucre & 0,156 & 0,153 & 1,253 & 1,085 \\
\hline Tolima & 0,598 & 0,555 & 1,067 & 1,053 \\
\hline Valle & 3,710 & 2,944 & 0,838 & 0,618 \\
\hline
\end{tabular}

Fuente: cálculos del autor con base en los datos del modelo CEER. 